\title{
Dark Matter Searches with HAWC
}

\author{
Corresponding authors: Joseph Lundeen*1 \\ for the HAWC Collaboration ${ }^{\dagger}$ \\ ${ }^{1}$ Michigan State University, Los Alamos National Laboratory \\ $\dagger$ https://www.hawc-observatory.org/collaboration/icrc2019.php \\ E-mail: lundeenj@msu.edu
}

The High Altitude Water Cherenkov Observatory (HAWC) detector has conducted a series of searches for very-high energy gamma-ray signals originating from particle dark matter. HAWC's wide $(\approx 2 \mathrm{sr}$ ) field of view allows it to search for emission from extended objects such as the M31 galaxy, the Virgo Cluster, and a region of the Galactic Halo. The daily coverage of $2 / 3$ of the sky of HAWC has been used to perform combined analyses of both the dwarf spheroidal and dwarf irregular galaxies. This archival data has also been used for a joint analysis of the dwarf spheroidal galaxies in combination with the Fermi-LAT, HESS, MAGIC and VERITAS experiments. New energy estimation algorithms have also enabled HAWC to search for and constrain gamma-ray line emission from dark matter. We will present the latest results from each of these dark matter analyses, which include some of the strongest limits on dark matter decay.

36th International Cosmic Ray Conference -ICRC2019-

July 24th - August 1st, 2019

Madison, WI, U.S.A.

${ }^{*}$ Speaker. 


\section{Introduction}

Astrophysical evidence for dark matter is plentiful, yet no particle candidate has yet been identified

\section{Indirect Dark Matter Detection}

Under the WIMP hypothesis, dark matter can produce gamma-rays through several mechanisms. One is through annihilation of two dark matter particles into Standard Model particles, described in the following equation:

$$
\frac{d \Phi}{d E}=\frac{J\langle\sigma v\rangle}{8 \pi M^{2}} \frac{d N(M, \text { channel })}{d E}
$$

where $\langle\sigma v\rangle$ is the velocity-weighted annihilation cross section and $M$ is the dark matter mass. The $J$-factor is defined as:

$$
J=\iint \rho_{d m}^{2}(l, \Omega) d l d \Omega
$$

an integral of the squared dark matter mass density profile over the line of sight and solid angle of the observation.

Is is also possible for a single dark matter particle to decay to a pair of standard model particles. The resulting gamma-ray spectrum is described by:

$$
\frac{d \Phi}{d E}=\frac{1}{\tau} \frac{D}{4 \pi M} \frac{d N(M, \text { channel })}{d E}
$$

where $\tau$ is the dark matter particle lifetime and $M$ is again the dark matter mass. The $D$-factor is defined as:

$$
D=\iint \rho_{d m}(l, \Omega) d l d \Omega,
$$

analogous to the J-factor, but with only one power of the density profile.

For both the continuous spectra, $\frac{d N}{d E}$ is the gamma-ray spectrum from a single dark matter annihilation [1]. The analyses presented here use PYTHIA 8.2 to calculate this function by simulating dark matter annihilation and recording the number of gamma rays produced. The spectra tested here assume $100 \%$ branching ratios into a given channel [2]. An example of dark matter spectral shapes, showing the characteristic hard energy cut-off at the dark matter mass, is shown in Fig. 1.

It is also possible for WIMPS to annihilate directly to gamma-ray pairs. This process would manifest as a sharp peak in the gamma-ray spectrum known as a gamma-ray line (see Fig. 1). Observing such a feature would be very strong evidence of annihilating dark matter, as this is the only process expected to produce lines at $\mathrm{TeV}$ energies. The location of the line also immediately reveals the dark matter particle mass.

\section{The HAWC Detector}

The High Altitude Water Cherenkov (HAWC) detector is a gamma-ray observatory located at Sierra Negra, Mexico. Consisting of an array of 300 water Cherenkov detectors and covering an area of $22,000 \mathrm{~m}^{2}$, it is able to detect gamma rays by the air showers they produce in the atmosphere. 


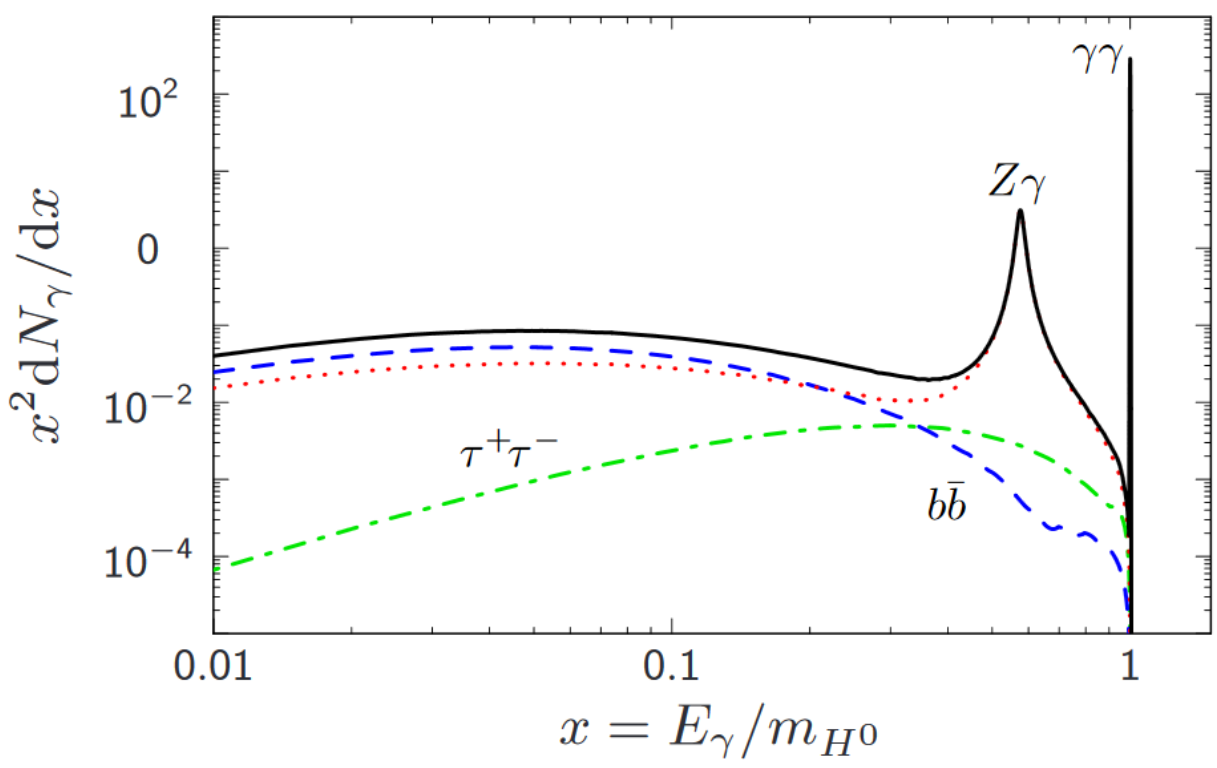

Figure 1: Example dark matter energy spectra for three continuum annihilation channels $\left(b \bar{b}, \tau^{+} \tau^{-}\right.$, and $Z \gamma$ ), as well as line emission directly to gamma-rays. This figure is taken from Fig. 1 of Ref. [3].

Air showers are created when charged particles or gamma rays interact with Earth's atmosphere, producing cascades of lower-energy particles. HAWC detects these secondary particles by the Cherenkov light they produce while passing through the water Cherenkov detectors, which is then detected with four photomultiplier tubes (PMTs) mounted at the bottom. Timing information is then used to reconstruct angle of arrival, and the distribution of charges is used to estimate energy [4].

The majority of air showers detected by HAWC come from charged cosmic rays. Therefore, cuts are applied to separate the hadronic events from the gamma rays before the data is analyzed. With cuts, 99\% of hadronic events are rejected at the highest energies [4]. However, the relative abundance of charged cosmic rays to gamma rays means the data is still dominated by charge cosmic rays after cuts. To estimate the remaining background, we use a technique called direct integration (DI). Events are integrated within some time period in hour angle of a point to estimate the associated background [4]. The signal at each point in the sky is then the number of excess gamma rays above this estimated background level..

HAWC is sensitive to gamma rays above $500 \mathrm{GeV}$ and is well-suited for detecting signals from multi-TeV dark matter masses. In addition, HAWC operates on a near-continuous duty cycle with a wide field-of-view that makes it ideal for performing survey-style observations [4].

HAWC uses a forward folding analysis to reconstruct energy spectra. Monte Carlos is used to simulate the HAWC detector response to a given energy spectrum, and the observed response is then mapped back to the best-fit true spectrum using maximum likelihood methods [4]. Several binning schemes are used. In the first, events are binned by $f_{\text {hit }}$, the fraction of available PMTs triggered by a shower. This technique is used for the majority of dark matter searches shown here. More recent algorithms have allowed HAWC to bin events in reconstructed energy as well by using 
the lateral charge distribution of a shower across the array. Two algorithms have been developed; one using a direct fit to the lateral charge distribution and the other using a neural network [5]. Both techniques give relatively consistent reconstructed spectra for known sources [5].

The analyses present here focus on searching for dark matter flux from known halos. Astrophysical evidence is used to estimate the J and D-factors, and HAWC data is searched for gammaray excesses consistent with dark matter spectra shapes. If no significant excess is found, limits are set on dark matter annihilation and decay.

\section{HAWC Dark Matter Searches}

\subsection{Dwarf Spheroidal Galaxies}

The dwarf spheroidal galaxies in the vicinity of the Milky Way are a set of faint halos, several of which contain relatively high dark matter content. The relatively sparse stellar population means there are few possible sources of gamma rays from these halos besides dark matter interactions.

This analysis looked at 15 dwarf spheroidal galaxies within the HAWC field of view. The J-factors were calculated using the CLUMPY package are summarized along with the sources in Table. 1 of Ref. [6]. A combined likelihood technique was used to produce a joint limit using data from all 15 dwarf spheroidal galaxies [6]. The combined limits for a select set of annihilation channels are shown in Fig. 2. A further join analysis is that combines data from the HAWC, Magic, Veritas, Hess, and Fermi Experiments [7] and is expected to yield even stronger limits.

\subsection{The Galactic Halo}

The Galactic halo is the closest known large dark matter halo, and has a correspondingly high expected gamma-ray flux from dark matter interactions. However, the unconstrained behavior of the dark matter density profile towards the center introduces a large systematic on the flux and the corresponding limits [8]. HAWC searched a highly extended region of the Galactic halo that was far enough away from the center to mitigate this systematic, which still yielding fairly strong limits on the flux. The limits for the more optimistic Einasto [9] [10] profile are shown as part of Fig. 2. A more optimized version of this analysis that will take into account the HAWC sensitivity in order to select an ideal region to search is currently in progress [11].

\subsection{M31}

The M31 (Andromeda) galaxy is another known sources of dark matter. M31 is a highly extended source with an angular radius of approximately 7 degrees. HAWC is able to use its wide field of view to search for emission from this large region. Three possible models are considered, assuming different concentrations of substructure [12]. For these proceedings, we summarize only the median case.

The constraints on dark matter decay for M31 are the strongest currently available for masses between $25 \mathrm{TeV}$ and $100 \mathrm{TeV}$ for the $b \bar{b}$ channel and are summarized in Fig. 2

\subsection{The Virgo Cluster}

The Virgo Cluster is an extended source consisting of two component halos, M87 and M49. The combined extent is approximately 3.6 degrees (full width at half maximum), making HAWC's 

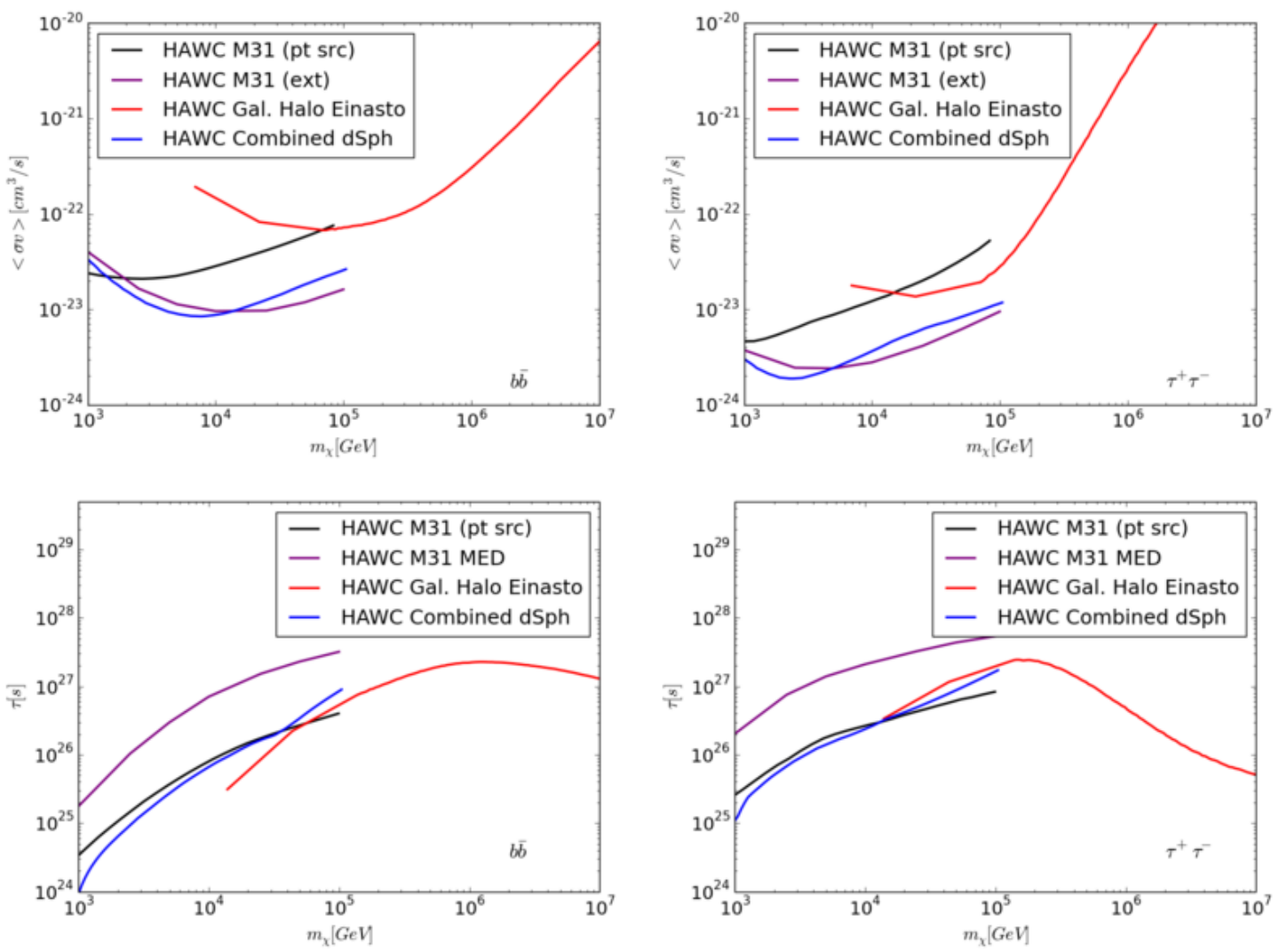

Figure 2: Dark matter annihilation and decay limits from the dwarf spheroidal galaxies [6], M31 galaxy [12], and Galactic halo [11] region into the $b \bar{b}$ and $\tau^{+} \tau^{-}$channels. Note that the Galactic halo limits assume the more concentrated Einasto profile.

wide field of view ideal for probing its emission [13]. The expected flux from the Virgo Cluster is highly dependent on the assumed contribution from sub-structure. Like the M31 galaxy, three possible models are, assuming different concentrations of substructure [13]. For these proceedings, we again summarize only the median case.

The lower limits on dark matter decay derived from the Virgo Cluster are the most constraining currently available for several dark matter masses and decay channels. Fig. 3 summarizes these limits for a set of channels and masses, along with comparisons to limits from other experiments.

\subsection{Gamma-Ray Lines}

The development of new energy estimation algorithms has allowed HAWC to search for gamma-ray lines. We search for gamma-ray lines originating from the dwarf spheroidal galaxies, using 1038 days of HAWC data. This search uses the ground parameter energy estimator to bin events in reconstructed energy space. A forward folding analysis is still used to map the reconstructed spectra to true spectra [4].

No significant excess was found for line emission from the dwarf spheroids, and upper limits are again set on $\langle\sigma v\rangle$. Fig. 4 shows the combined likelihood upper limits for all dwarf spheroidals 

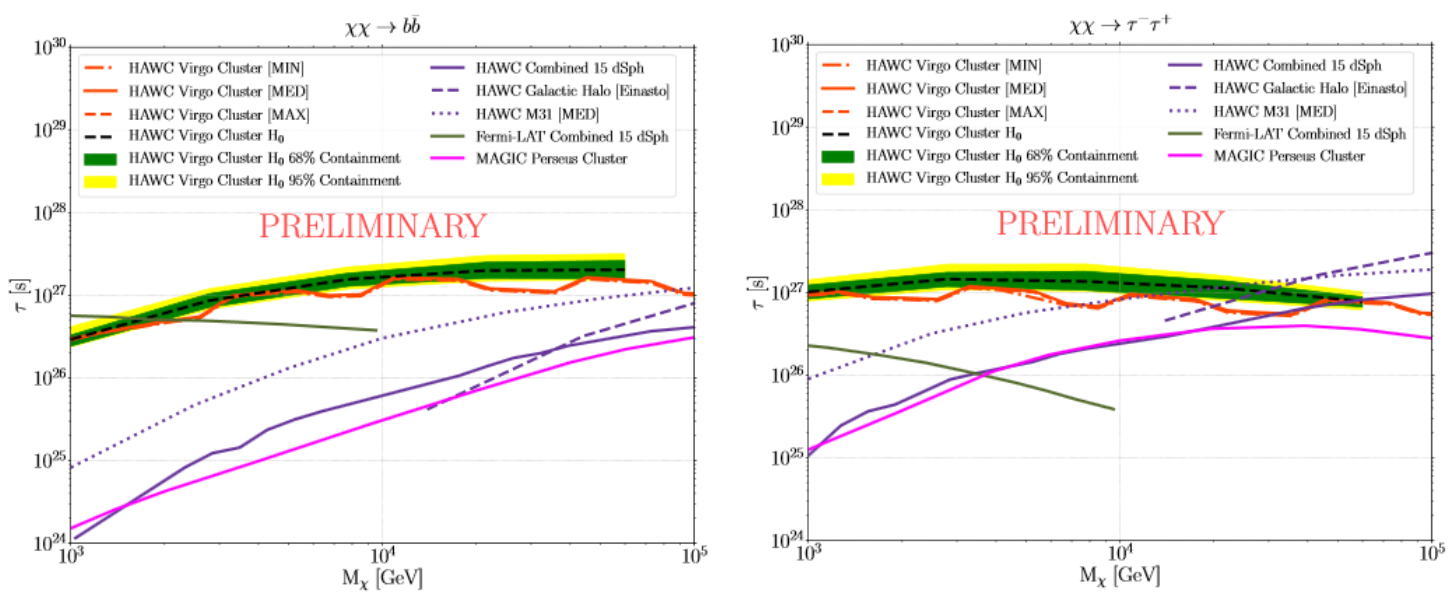

Figure 3: Dark matter annihilation limits from the Virgo cluster into the $b \bar{b}$ and $\tau^{+} \tau^{-}$channels [13]. For masses above $2 \mathrm{TeV}$, the $b \bar{b}$ limits are the most constraining available, while the $\tau^{+} \tau^{-}$limits are the most constraining for masses below $5 \mathrm{TeV}$.

considered, compared to limits set by other experiments. The HAWC limits are the most constraining above $10 \mathrm{TeV}$, and the only currently available constraints above $20 \mathrm{TeV}$.

\subsection{Dwarf Irregular Galaxies}

Additionally, the dwarf irregular galaxies are candidates for dark matter emission. Due to their non-spheroidal shape, calculating the J-factors requires techniques documented in Ref. [14]. Again, no significant excess is found and upper limits are set (Fig. 5) [15].

\section{Conclusion}

HAWC has been able to probe a diverse set of potential dark matter sources due to its surveystyle view of the sky and wide field-of-view. Joint analyses of the dwarf spheroidal galaxies have produced strong constraints on dark matter interactions, and further collaboration with other gamma-ray experiments is expected to improve these limits. Additional analyses have constrained dark matter emission from the M31 galaxy, dwarf irregular galaxies, and the Galactic halo, with improvements currently in development on the later. Newly developed energy estimation algorithms have allowed HAWC to search for and constrain gamma-ray line emission from dark matter. Searches of the M31 galaxy and the Virgo Cluster have yielded some of the most constraining limits on dark matter decay currently available. Continuous data-taking with HAWC will only improve these results and allow it to continue searching for dark matter in the gamma-ray regime.

\section{References}

[1] K. N. Abazajian and J. P. Harding, JCAP 1201 (2012) 041.

[2] T. SjÃústrand, S. Ask, J. R. Christiansen, R. Corke, N. Desai, P. Ilten, S. Mrenna, S. Prestel, C. O. Rasmussen, and P. Z. Skands, Comput. Phys. Commun. 191 (2015) 159-177. 


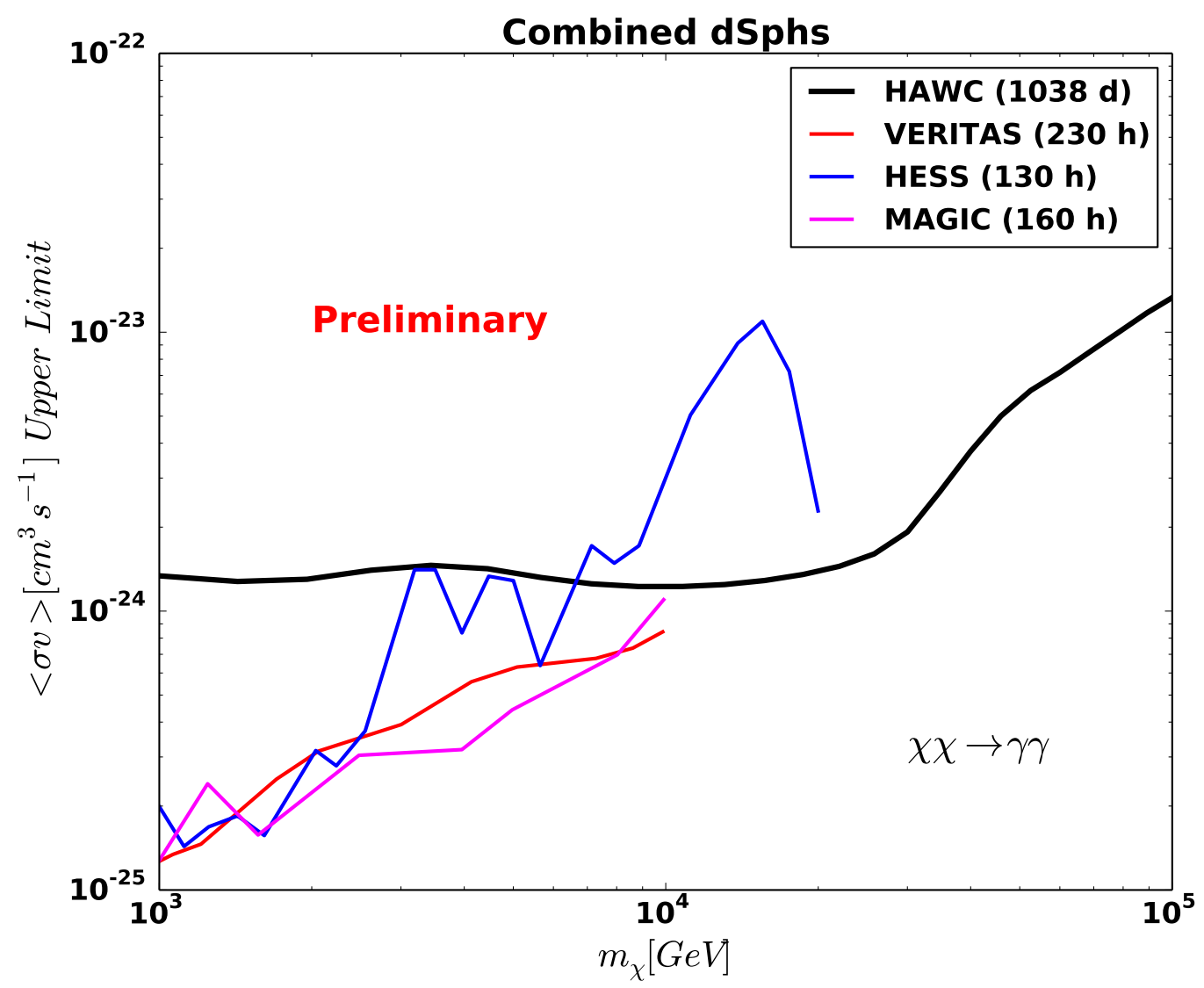

Figure 4: Combined upper limits on dark matter gamma-ray line emission from the dwarf spheroidal galaxies with HAWC, compared with upper limits from other experiments. The HAWC limits are the only constraints above $10 \mathrm{TeV}$.
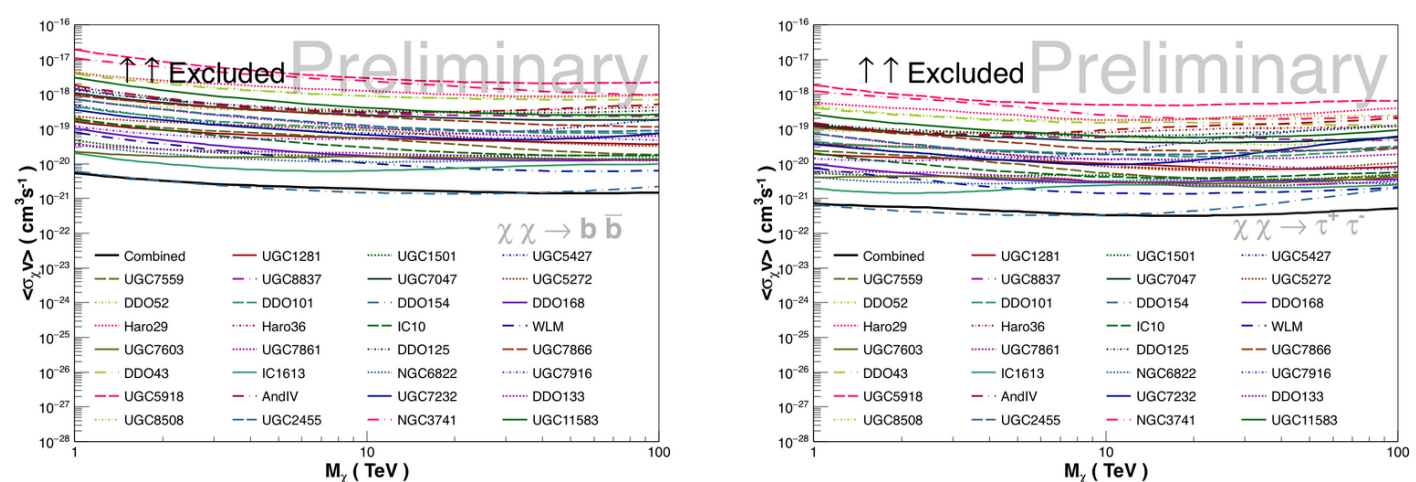

Figure 5: Dark matter annihilation limits from the dwarf irregular galaxies into the $b \bar{b}$ and $\tau^{+} \tau^{-}$channels [15]. 
[3] M. Gustafsson, E. Lundström, L. Bergström, and J. Edsjö, Phys. Rev. Lett. 99 (Jul, 2007) 041301.

[4] A. U. Abeysekara et al., Astrophys. J. 843 (2017) 39.

[5] HAWC Collaboration, A. U. Abeysekara et al., arXiv:1905.12518.

[6] A. Albert et al., Astrophys. J. 853 (2018) 154.

[7] J. P. Harding et al., Proceedings of Science (2019).

[8] HAWC Collaboration, A. U. Abeysekara et al., JCAP 1802 (2018) 049.

[9] J. Stadel, D. Potter, B. Moore, J. Diemand, P. Madau, M. Zemp, M. Kuhlen, and V. Quilis, Mon. Not. Roy. Astron. Soc. 398 (2009) L21-L25.

[10] J. F. Navarro, A. Ludlow, V. Springel, J. Wang, M. Vogelsberger, S. D. M. White, A. Jenkins, C. S. Frenk, and A. Helmi, Mon. Not. Roy. Astron. Soc. 402 (2010) 21.

[11] J. Lundeen et al.

[12] HAWC Collaboration, A. Albert et al., JCAP 1806 (2018) 043. [Erratum: JCAP1904,no.04,E01(2019)].

[13] T. Yapici et al., Proceedings of Science (2019).

[14] E. V. Karukes and P. Salucci, Mon. Not. Roy. Astron. Soc. 465 (2017) 4703-4722.

[15] S. Hernandez et al. 